\title{
3D Blood Flow Reconstruction from 2D Angiograms
}

\author{
Esther-S. Platzer ${ }^{1}$, Frank Deinzer $^{2}$, Dietrich Paulus ${ }^{3}$, Joachim Denzler $^{1}$ \\ ${ }^{1}$ Institut für Mathematik/Informatik, Friedrich-Schiller-Universität, 07743 Jena \\ ${ }^{2}$ Siemens AG, Medical Solutions, 91301 Forchheim \\ ${ }^{3}$ Institut für Computervisualistik, Universität Koblenz-Landau, 56070 Koblenz \\ platzer@informatik.uni-jena.de
}

\begin{abstract}
A method for 3-d blood flow reconstruction is presented. Given a 3-d volume of a vessel tree and a 2-d sequence of angiograms, the propagation information can be back-projected into the 3 -d vessel volume. In case of overlapping vessel segments in the 2 -d projections, ambiguous back-projection results are obtained. We introduce a probabilistic blood flow model for solving these ambiguities. Based on the last estimated state and known system dynamics the next state is predicted, and predictions are judged by the back-projected information. The discrete realization is done with a particle filter. Experiments prove the efficiency of our method.
\end{abstract}

\section{Introduction}

In the field of vessel diagnostics, 2-d digital subtraction angiographies (DSA) and 3-d reconstructions of the vessel trees belong to the state of art. Since a 2-d DSA only provides 2- $\mathrm{d}+\mathrm{t}$ information and a 3-d reconstruction offers nontemporal information about the vessel morphology, a fusion of both is necessary to visualize spatial and temporal coherences.

Establishing a 2-d/3-d mapping allows the look up of the bolus arrival time (BAT) for every voxel from the 2-d angiograms. Unfortunately, the mapping between 2-d pixels and 3-d voxels is usually ambiguous due to the loss of information caused by dimensionality reduction. State of the art work offers several ways to establish the mapping between pixels and voxels. Schmitt et. al [1], for example, cluster the 3-d vessel tree by a region growing algorithm and look up the BAT for every cluster in the 2-d angiograms by projecting the clusters to the detector plane. In case of ambiguities, correction of the cluster BAT with help of a median filter and heuristic assumptions is done. In [2] the mapping is established by artificial projections of the 3 -d volume, which allows a 2-d/2-d mapping between angiogram and artificial projection. Ambiguities are solved for by the use of a bi-plane angiography system, associated with an increased exposure dose for patients. A third approach are numerical flow simulations, also called computational fluid dynamics (CFD). They simulate the blood flow through a given 3-d model of the vessel tree based on physics and the navier-stokes equations [3]. This simulation is very time-consuming and has to be done for every 
3-d-model individually. Under use of actual hardware, this is not acceptable for clinical course of life.

We present a probabilistic model of blood flow for dissolving ambiguous mappings based on recursive state estimation. The blood flow model evaluates the probability of the back-projection results with simultaneous consideration of the existing knowledge from previous time steps. So uncertainty is decreased by the integration of additional knowledge in every new time step.

\section{Materials and Methods}

Our goal is to create a probabilistic model of the 3-d blood flow. With the knowledge we get from the 2-d angiograms, modelling was done by a Bayesian filter that estimates the non-measurable state $\boldsymbol{q}_{t}$ of a system based on the observation $\boldsymbol{o}_{t}$ and the actual belief of the previous state. $\langle\boldsymbol{o}\rangle_{t}$ is the observation sequence of all observations made from $t=0$ until now:

$$
p\left(\boldsymbol{q}_{t} \mid\langle\boldsymbol{o}\rangle_{t}\right)=\underbrace{p\left(\boldsymbol{o}_{t} \mid \boldsymbol{q}_{t}\right)}_{\text {evaluation }} \int \underbrace{p\left(\boldsymbol{q}_{t} \mid \boldsymbol{q}_{t-1}\right)}_{\text {prediction }} \underbrace{p\left(\boldsymbol{q}_{t-1} \mid\langle\boldsymbol{o}\rangle_{t-1}\right)}_{\text {recursion }} d \boldsymbol{q}_{t-1}
$$

For non-linear and non-gaussian, multimodal state estimation problems normally no analytic, closed-form solution exists. Therefore a discrete realization was done using a particle filter $[4,5]$ in combination with the Condensation algorithm [6].

Given an initial belief of the fill state of the vessel tree and assumptions on the actual velocity and direction of the bolus front, a prediction of the covered vessel distance in the next step is possible. In the discrete case, this motion model is based on a uniform weighted voxel graph [7]. The costs of edge traversal from one voxel to another represent the distances in the vessel tree, which are weighted by a gaussian distribution with a mean corresponding to the actual velocity. For every particle, its new position can be predicted under use of a shortest-path algorithm.

The evaluation step is performed with help of sensor data, called observation $\boldsymbol{o}$, where $\boldsymbol{o}_{t}$ is represented by the extracted and back-projected flow information from the 2-d angiogram at time $t$. Particle weighting is done accordingly to the back-projected flow information and results in a corrected belief at time $t$.

By taking the corrected estimate as initial belief of the next time step, the gathered knowledge is propagated and increased over time. Practically, this knowledge propagation is done by resampling from the previous particle set accordingly to the particle weights.

Because no a priori knowledge is given for the initial state, it is modeled by a uniform distribution. A robust estimation in the first time steps is not possible. This will become clear in Fig. 3. Given Fig. 4(b) and a uniform particle distribution in Fig. 4(c), particles in the left and right vessel ends would gain a good evaluation in the first steps, resulting in a propagation of flow in both vessel arms. Smoothing-filters [8] allow the evaluation of a density on past and future 
observations. They are motivated by the fact, that the density $p\left(\boldsymbol{q}_{T} \mid\langle\boldsymbol{o}\rangle_{T}\right)$ contains all information from all angiograms and can be described by the following formula, where $\langle\boldsymbol{o}\rangle_{T}$ denotes all observations from angiograms 0 till $T$ :

$$
p\left(\boldsymbol{q}_{t} \mid\langle\boldsymbol{o}\rangle_{T}\right) \propto \underbrace{p\left(\boldsymbol{q}_{t} \mid\langle\boldsymbol{o}\rangle_{1: t}\right)}_{\text {Forward Filter Backward Filter }} \underbrace{p\left(\langle\boldsymbol{o}\rangle_{t+1: T} \mid \boldsymbol{q}_{t}\right)}_{\text {B }}
$$

The use of a forward-backward smoother in combination with Condensation is described in [9]. The forward part of the filter is given by a simple Condensation run, resulting in a robust estimate of $\boldsymbol{q}_{T}$. Using this estimate as initial belief of an analogue backward run eliminates ambiguous estimates and returns a good estimate of the initial state. Usually, in the prediction step of the backward-filter, state changes are restricted to the states predetermined by the forward step:

$$
p\left(\langle\boldsymbol{o}\rangle_{t+1: T} \mid \boldsymbol{q}_{t}\right)=\int p\left(\langle\boldsymbol{o}\rangle_{t+1: T} \mid \boldsymbol{q}_{t+1}\right) p\left(\boldsymbol{q}_{t+1} \mid \boldsymbol{q}_{t}\right) d \boldsymbol{q}_{t+1}
$$

In our work, we changed this restriction and used the same motion model as in the forward step, in order to compensate for the failure of observations in a sequence of 2-d-angiograms, caused by vessel overlaps. (3) changes to:

$$
p\left(\langle\boldsymbol{o}\rangle_{t+1: T} \mid \boldsymbol{q}_{t}\right)=\int p\left(\langle\boldsymbol{o}\rangle_{t+1: T} \mid \boldsymbol{q}_{t+1}\right) p\left(\boldsymbol{q}_{t} \mid \boldsymbol{q}_{t+1}\right) d \boldsymbol{q}_{t+1}
$$

In the evaluation step, a correction of the gained particle weights of the forwardstep is performed:

$$
p\left(\langle\boldsymbol{o}\rangle_{t: T} \mid \boldsymbol{q}_{t}\right)=p\left(\boldsymbol{o}_{t} \mid \boldsymbol{q}_{t}\right) p\left(\langle\boldsymbol{o}\rangle_{t+1: T} \mid \boldsymbol{q}_{t}\right)
$$

In case of vessel bifurcations, the underlying densities become multimodal and two bolus fronts have to be propagated. At vessel ends, the underlying densities are unimodal, even if in-between vessel bifurcations enforce multimodal densities. In the forward step, the split-up of blood flow is supported by the motion model, but in the backward step the propagation of particles into the bifurcated vessel segments is restricted due to inverse traversal of the vessel tree. A schematic illustration of this problem is given in Fig. 1, where a particle propagation at

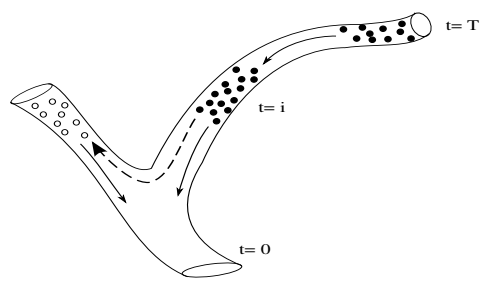

Fig. 1. Restriction of the change from an unimodal (black) to a multimodal (outlined) density. The distance in the graph is marked with the dashed line 
$t=i$ into the left vessel segment is prohibited because of too large distances. This problem was solved by an additional, slightly modified forward run, offering the data for a correct visualization. It relies on the robust estimate of the initial state, returned by the forward-backward smoother and uses $\langle\boldsymbol{o}\rangle_{t}$ instead of $\boldsymbol{o}_{t}$ for the likelihood estimation in order to compensate for the different velocities in the bifurcated vessel segments.

\section{$3 \quad$ Experiments and Results}

Evaluation was done on different angiography sequences of a vessel phantom, including views resulting in many overlaps, especially at beginning and the end of the vessels (Fig. 3 (a)-(b)). Fig. 4(c) shows the 3-d reconstruction of the vessel phantom. All acquisitions were done on a Siemens Artis dFA, and for all computations an AMD Athlon64 X2 3800+ was used. Computation time for one time step with 15000 particles lies at approximately 30-40 seconds were most time is spent on the evaluation process. All experiments were performed with differentsized particle sets ranging from 1000 till 15000 particles. We compared our results to those of pure back-projection. Additionally, for ground-truth evaluation, the ambiguous back-projection information was solved for manually, and the number of correct particles regarding a non-ambiguous flow was determined. In all experiments a particle size of 2000 copes a feasible 3 -d blood flow reconstruction. All ambiguities could be dissolved, even in case of overlapping vessel segments at the beginning of the sequence. Furthermore, the failure of observations can be compensated due to the estimation process based on the system dynamics. Observation failures result from artefacts avoiding a correct extraction of flow information, as well as huge vessel overlaps that cause mutual subtraction of the flow information (Fig. 4(b)). Fig. 2 shows the number of correct particles out of all good-evaluated particles reconstructed from Fig. 4(b). In the first forward run (Fig. 2(a)), the number of correct particles (shaded) is less than that of all good-evaluated particles (solid), whereas in the second forward run (Fig. 2(b)) all ambiguities could be resolved. Snapshots of the 3-d reconstruction and its visualization of blood flow are shown in Fig. 3(d)-(f).

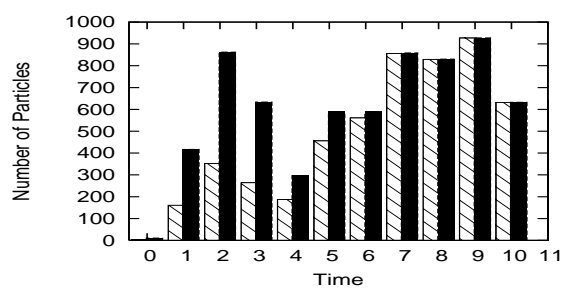

(a) first run

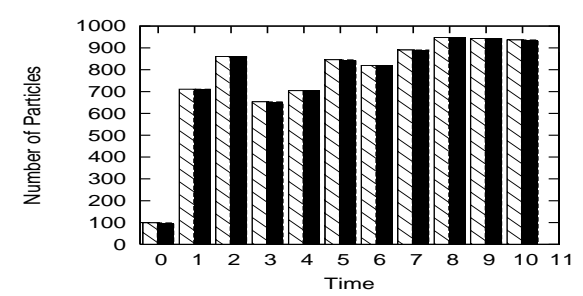

(b) second run

Fig. 2. Number of correct particles (shaded) related to the number of all good-evaluated particles (solid) for both forward runs (1000 particles) 
Fig. 3. Angiograms from frontal (a) and side view (b), 3-d reconstruction of the vessel phantom (c) and results of the 3-d blood flow visualization (d)-(f)

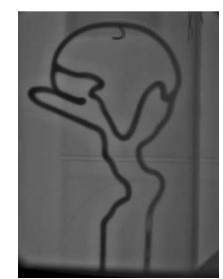

(a)

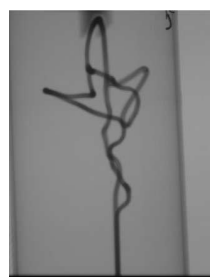

(b)

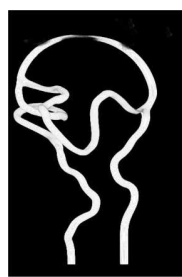

(c)

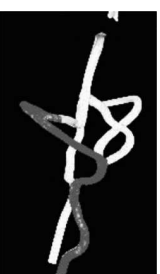

(d)

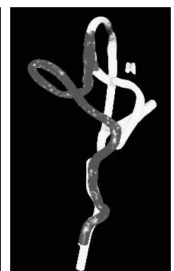

(e)

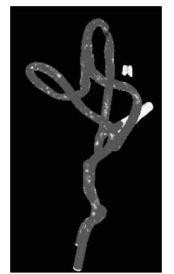

(f)

\section{Discussion}

A method for 3-d reconstruction of blood flow with help of the propagation information, gathered from a 2-d angiography sequence, was presented. Our probabilistic model relies on the theory of state estimation in a dynamic system, and discrete realization was done with a forward-backward-forward particle filter. Weak point of our model is the motion model. Because system dynamics not being stable, especially in case of vessel bifurcations, the motion model has to be updated during the whole estimation process. The computational overload could be reduced by the use of adaptive particle filters [5], and the motion model could be improved by CFD-methods which allow further modelling of non-observable flow characteristics in aneurysms or the diffusion of blood and contrast agent.

\section{References}

1. Schmitt H, Grass M, Rasche V, et al. An X-ray-based method for the determination of the contrast agent propagation in 3-d vessel structures. IEEE Trans Med Imaging. $2002 ; 21(3): 251-62$.

2. Hüllmandel T. Räumliche und zeitliche Rekonstruktion in der Neuroradiologie [Diplomarbeit]. Universität Würzburg; 2004.

3. Ford MD, Stuhne GR, Nikolov HN, et al. Virtual angiography for visualization and validation of computational models of aneurysm hemodynamics. IEEE Trans Med Imaging. 2005 12;24(12):1586-92.

4. Arulampalam S, Maskell S, Gordon N, et al. A tutorial on particle filters for on-line non-linear/non-Gaussian Bayesian tracking. IEEE Trans Signal Process. $2002 ; 50(2): 174-88$.

5. Doucet A, de Freitas N, Gordon N. Sequential Monte Carlo Methods in Practice. Springer, Berlin; 2001.

6. Isard M, Blake A. CONDENSATION: Conditional density propagation for visual tracking. Int J Computer Vis. 1998;29(1):5-28.

7. Xu L. Konzipierung und Realisierung eines interaktiven Editors für Gefäßgraphen. DKFZ Heidelberg; 2005.

8. Kitagawa G. Monte Carlo filter and smoother for non-Gaussian nonlinear state space models. J Comput Graph Stat. 1996;5(1):1-25.

9. Isard M, Blake A. A smoothing filter for Condensation. Proc ECCV. 1998;1:767-81. 